\title{
Do mixed histological features affect survival benefit from neoadjuvant platinum-based combination chemotherapy in patients with locally advanced bladder cancer? A secondary BJUI analysis of Southwest Oncology Group-Directed
Intergroup Study (S8710)
}

Emil Scosyrev' ${ }^{1}$ Benjamin W. Ely ${ }^{2}$, Edward M. Messing ${ }^{1}$, V.O. Speights ${ }^{3}$, H. Barton Grossman ${ }^{4}$, David P. Wood ${ }^{5}$, Ralph W. de Vere White ${ }^{6}$, Nicholas J. Vogelzang ${ }^{7}$, Donald L. Trump ${ }^{8}$, Ronald B. Natale ${ }^{9}$, Catherine M. Tangen ${ }^{2}$, E. David Crawford ${ }^{10}$ and lan M. Thompson ${ }^{11}$ 'University of Rochester, Rochester, NY, ${ }^{2}$ Southwest Oncology Group Statistical Center, Seattle, WA, ${ }^{3}$ Scott and White Clinic, Temple, TX, ${ }^{4}$ M.D. Anderson Cancer Center, Houston, TX, ${ }^{5} U$ niversity of Michigan, Ann Arbor, MI, ${ }^{6}$ University of California at Davis, Sacramento, CA, ${ }^{7}$ Comprehensive Cancer Centers of Nevada, Las Vegas, NV (Southwest Oncology Group and previously, Cancer and Leukemia Group B), ${ }^{8}$ Roswell Park Cancer Institute, Buffalo, NY (Eastern Cooperative Oncology Group), ${ }^{9}$ Cedars-Sinai Comprehensive Cancer Center, Los Angeles, $C A$, and ${ }^{10}$ University of Colorado, Aurora, CO, and "University of Texas Health Science Center, San Antonio, TX, USA

Accepted for publication 1 September 2010

Study Type - Therapy (RCT) Level of Evidence $1 b$

\section{OBJECTIVE}

- To determine whether the effect of neoadjuvant chemotherapy with methotrexate, vinblastine, doxorubicin and cisplatin (MVAC) on the survival of patients with locally advanced urothelial carcinoma (UC) of the bladder treated with radical cystectomy varies with the presence of nonurothelial components in the tumour.

\section{PATIENTS AND METHODS}

- This is a secondary analysis of the Southwest Oncology Group-directed

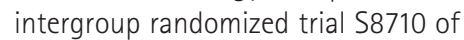
neoadjuvant MVAC followed by cystectomy versus cystectomy alone for treatment of locally advanced UC of the bladder.

- For the purpose of these analyses, tumours were classified based on the presence of non-urothelial components as either pure UC $(n=236)$ or mixed tumours ( $n$ =59). Non-urothelial components included squamous and glandular differentiation.

- Cox regression models were used to estimate the effect of neoadjuvant MVAC on all-cause mortality for patients with pure UC

What's known on the subject? and What does the study add?

In a meta-analysis of randomized trials, neoadjuvant platinum-based combination chemotherapy administered before definitive local treatment improved survival of patients with muscle-invasive bladder cancer compared with definitive local treatment alone. However, it was not known whether chemotherapy was equally effective for pure urothelial carcinoma versus urothelial carcinoma with mixed histological features, such as squamous or glandular differentiation.

To address this question we performed a secondary analysis of the Southwest Oncology Group-directed intergroup randomized trial S8710 of neoadjuvant methotrexate, vinblastine, doxorubicin, and cisplatin (MVAC) followed by cystectomy versus cystectomy alone for treatment of locally advanced urothelial cancer of the bladder. Our findings suggest that presence of squamous or glandular differentiation does not confer resistance to combination chemotherapy with MVAC and in fact may be an indication for the use of neoadjuvant chemotherapy before radical cystectomy.

and for patients with mixed tumours, with adjustment for age and clinical stage.

\section{RESULTS}

- There was evidence of a survival benefit from chemotherapy in patients with mixed tumours (hazard ratio 0.46; 95\% Cl 0.25$0.87 ; P=0.02)$. Patients with pure UC had improved survival on the chemotherapy arm but the survival benefit was not statistically significant (hazard ratio 0.90; $95 \% \mathrm{Cl}$

$0.67-1.21 ; P=0.48$ ).

- There was marginal evidence that the survival benefit of chemotherapy in patients with mixed tumours was greater than it was for patients with pure UC (interaction $P=0.09)$.

\section{CONCLUSION}

- Presence of squamous or glandular differentiation in locally advanced UC of the bladder does not confer resistance to MVAC and in fact may be an indication for the use of neoadjuvant chemotherapy before radical cystectomy.

\section{KEYWORDS}

urothelial carcinoma, mixed histological features, neoadjuvant chemotherapy 


\section{INTRODUCTION}

Bladder cancer $(\mathrm{BC})$ is the fifth most commonly diagnosed malignancy in the USA, with more than 70000 new cases and more than 14000 deaths reported in 2009 [1,2]. Most deaths from BC occur among patients with an initial diagnosis of muscle-invasive disease (stages T2-T4). Standard therapy for resectable (T2-T4a) muscle-invasive $\mathrm{BC}$ without known metastases includes radical cystectomy with pelvic lymphadenectomy [3]. Unfortunately, many patients with apparently resectable muscle-invasive $B C$ have undiagnosed micrometastatic disease at the time of definitive surgery. In a series of 1054 patients treated with radical cystectomy and pelvic lymphadenectomy between 1977 and 1997, with a median follow up of 10.2 years, $\mathrm{BC}$ recurred in 311 patients (30\%) with a median time to recurrence of 12 months. Three-quarters of all patients with disease recurrence had distant metastases [4].

Early treatment of micrometastatic disease with neoadjuvant platinum-based combination chemotherapy (PBCC) administered before definitive local treatment (cystectomy and/or radiotherapy) has been compared with local treatment alone in several randomized trials. A meta-analysis of these trials showed that addition of a neoadjuvant PBCC regimen to local treatment improves the average 5 -year survival by $5 \%$ on the additive scale (from 45\% to 50\%) [5]. Several trials also reported that the use of neoadjuvant PBCC may increase the probability of pathological stage zero (pT0) at cystectomy from approximately $12-15 \%$ in the cystectomy-only arm to $33-38 \%$ in the PBCC plus cystectomy arm $[6,7]$.

Although most patients who are treated with radical cystectomy for muscle-invasive $B C$ have pure urothelial carcinoma (UC), tumours with mixed histological features (UC coexisting with non-urothelial histology) are also common. For example, in a series of 243 patients with clinical stage of at least T2, 96 patients (40\%) had mixed histological features, most frequently UC with squamous and/or glandular differentiation [8].

It is currently unknown whether the benefit of neoadjuvant PBCC on pathological downstaging and survival of patients with apparently resectable muscle-invasive UC treated with radical cystectomy is influenced by the presence of a non-urothelial component in the tumour. Observational studies suggested that among patients with metastatic $B C$, patients with pure UC as well as patients with mixed tumours can achieve a complete clinical response to PBCC (disappearance of all clinical and radiographic evidence of disease) $[9,10]$. In one series of patients with metastatic $B C$, complete clinical response to $\mathrm{PBCC}$ was reported in $39 \%$ of 74 patients with pure UC and in $25 \%$ of 20 patients with mixed histology (UC with squamous, glandular or spindle cell components) [9]. In another series of patients with metastatic UC, any clinical response to PBCC (complete or partial) was observed in $44 \%$ of 389 patients with pure UC and in 34\% of 42 patients with mixed urothelial and squamous histology [10].

These findings reported for metastatic UC may not be directly applicable to patients with apparently resectable $\mathrm{BC}$ because the biology of metastatic and locally advanced tumours may be different. In particular, complete clinical response of metastatic lesions (which is determined primarily by imaging studies) may not be equivalent to complete pathological response of the primary tumour in the bladder (stage pT0 at cystectomy). In addition, survival of patients with metastatic $\mathrm{BC}$ is usually very poor. While histological type may not have a strong impact on survival of patients with this very advanced form of disease, it may theoretically influence both response to chemotherapy and survival of patients with less advanced tumours treated with cystectomy and neoadjuvant PBCC with curative intent.

The purpose of the current study is to determine whether the effect of neoadjuvant PBCC on pathological down-staging and survival of patients with locally advanced UC of the bladder treated with radical cystectomy was influenced by the presence of non-urothelial components in the tumour.

\section{PATIENTS AND METHODS}

This is a secondary analysis of the Southwestern Oncology Group (SWOG) directed intergroup trial of neo-adjuvant methotrexate, vinblastine, doxorubicin, cisplatin (MVAC) followed by cystectomy versus cystectomy alone (SWOG 8710) [6]. Eligibility criteria for the trial included clinical stage T2-T4a NO MO UC of the bladder, no previous pelvic radiation, adequate renal, hepatic and haematological function, and a SWOG performance status of 0 or 1 [6]. A total of 307 eligible patients were enrolled between 1987 and 1998 and they were randomized to either MVAC plus cystectomy $(n=153)$ or cystectomy alone ( $n=154$ ) (Fig. 1). According to the study protocol, two pathological reviews were planned for each patient: first, a central pathological review of the pre-registration biopsy (transurethral resection) specimen to confirm eligibility, and second, a review of the cystectomy specimen to determine the pathological stage. As was discussed in the original publication, the first review was not performed for 46 patients because slides were not submitted or were lost in shipment [6]. These patients were enrolled in the trial and underwent randomization. Of the remaining patients, four had missing information on histological type from the first review (because of inadequate specimen submission). For these 50 patients $(46+4)$, histological type was determined from the institutional pathology reports. For all other patients, histological type was determined by the central pathological review (performed by a single expert genitourinary pathologist per case).

For the purpose of analyses reported in this paper, tumours were classified based on the presence of non-urothelial components as either pure UC $(n=236)$ or mixed tumours ( $n=61)$. For 10 patients, tumours could not be definitively classified as either pure or mixed based on available information. These 10 patients were excluded from our analysis. Non-urothelial components included squamous histology $(n=37)$, adenocarcinoma $(n=20)$, squamous histology with adenocarcinoma $(n=2)$ and other histological types $(n=2)$. The two patients with other histological types (one small cell and one signet ring) were also excluded to make the mixed histology group a more homogeneous pathological entity (UC with squamous and/or glandular differentiation). None of the tumours in this study had a documentation of micropapillary features. The relative frequencies of cases contributed by different study centres were similar for the two histology groups (pure UC and mixed tumours).

The primary covariates of interest in the current analyses were 'treatment' (MVAC plus cystectomy vs cystectomy alone) and 'histological type' (pure UC vs mixed tumours). Other candidate covariates 
FIG. 1. CONSORT diagram for the SWOG 8710 trial: MVAC, methotrexate, vinblastine, doxorubicin, and cisplatin; UC, urothelial carcinoma.

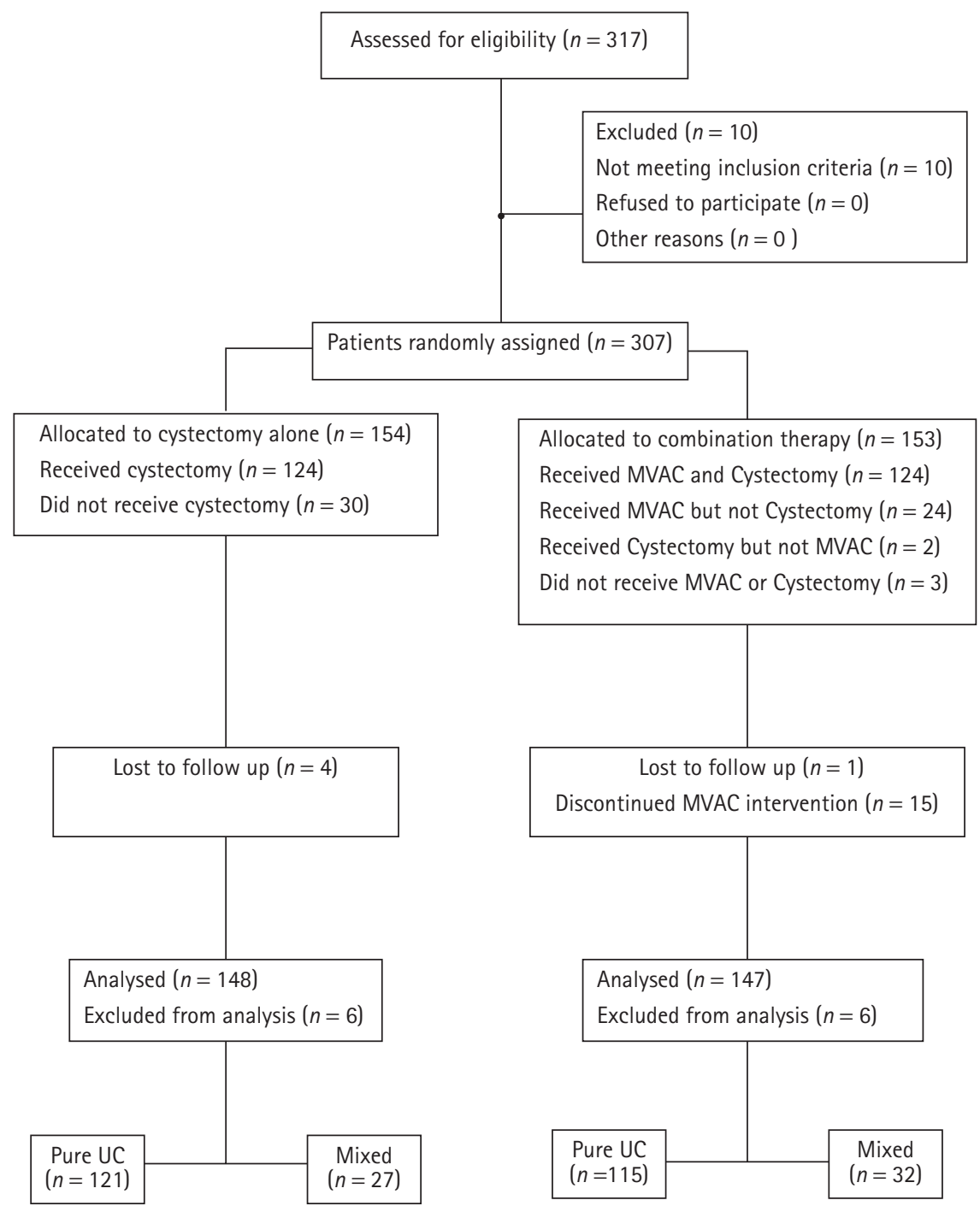

included age at randomization (in years), clinical stage (T2 vs T3-T4a), gender (male or female), and race (white or other race). The clinical stages were defined according to the fourth edition of the American Joint Committee on Cancer staging manual $[6,11]$. Two outcome measures were examined in the current study: the probability of no residual tumour in the cystectomy specimen (stage pTO at cystectomy), and the hazard of death from all causes (all-cause mortality). All-cause mortality was the primary endpoint of the trial according to the original study protocol. Survival time was measured from randomization until death from any cause. All patients provided written informed consent, and the study was approved by the ethics committees of participating institutions. tumours is different in magnitude from the effect of treatment in patients with pure UC. In the analysis of tumour down-staging, we made a conservative assumption that patients who did not undergo cystectomy, regardless of reason, had residual disease (they were considered not to have stage pTO).

The effect of MVAC on all-cause mortality was estimated separately for patients with mixed tumours and for patients with pure UC using the Cox model [15]. This model was also used to estimate the effect of histological type (mixed tumours vs pure UC) on all-cause mortality within each treatment arm and to test for treatment-by-histological type interaction. All regression models were stratified on clinical stage and included age as a continuous covariate. Hence, the estimated effects of treatment and histological type on the hazard of death from all causes were controlled for age and clinical stage at randomization in all comparisons reported in this paper. The assumptions of proportional hazards and of linearity of age (the only continuous covariate) with respect to the loghazard were checked and no major model violations were observed. Product terms were used to test for treatment-by-histological type interaction. All analyses were performed in SAS version 9.2 (SAS Institute, Cary, NC, USA). All reported $P$ values are two-sided.

\section{RESULTS}

The distribution of patient characteristics for each combination of treatment and histological type is shown in Table 1. Table 2 shows numbers and percentages of patients who had stage pTO based on pathological examination of the cystectomy specimen and numbers and percentages of patients whose pT0 status was not known. The pT0 status was known for 266 patients who either received cystectomy $(n=243)$ or had surgery cancelled or aborted because of overt disease progression/unresectable disease. The pTO status was not known for 29 patients who did not undergo cystectomy for reasons other than overt disease progression (e.g. refused cystectomy for personal reasons).

The percentages of patients with stage pTO in Table 2 were calculated by dividing the number of patients with pTO by the total number of patients in the corresponding combination of treatment with histological type, with the conservative assumption that 
all patients whose pT0 status was not known had residual disease (did not have stage pTO). In the crude analysis, the additive downstaging effect (ADE) of chemotherapy estimated with this conservative assumption was equal to $34 \%$ minus $4 \%$ (i.e. $30 \%$; $P=$ $0.004)$ for patients with mixed tumours, and $29 \%$ minus $14 \%$ (i.e. $15 \%$; $P=0.006$ ) for patients with pure UC. The estimated ADE of mixed histology was equal to $34 \%$ minus 29\% (i.e. $5 \% ; P=0.53$ ) in the MVAC-pluscystectomy arm, and 4\% minus 14\% (i.e. $-10 \%$; $P=0.20$ ) in the cystectomy-only arm.

The ADEs of treatment and histological type estimated with adjustment for clinical stage are shown in Table 3. These effects were very similar to the crude effects in terms of their magnitude and statistical significance.

Evidence of tumour down-staging to pT0 after chemotherapy was clearly present among patients with mixed tumours (ADE = $28 \%, P=0.004)$ and among patients with pure UC (ADE $=15 \%, P=0.004$; interaction $P=0.15)$.

Among patients with mixed tumours randomized to MVAC plus cystectomy, stage pT0 at the time of definitive surgery was observed in 6 of 20 patients with urothelial and squamous differentiation and in 5 of 10 patients with urothelial and glandular differentiation. Hence, pathological downstaging after chemotherapy was observed in both sub-types of mixed tumours, and was clearly not limited to only one sub-type (squamous or glandular).

Table 4 shows the estimated hazard ratios (HR) for the effect of MVAC on all-cause mortality among patients with mixed tumours (model 1) and among patients with pure UC (model 2). Also included in Table 4 are the estimated HR for the effect of mixed histology on all-cause mortality among patients randomized to MVAC-plus-cystectomy (model 3) and among patients randomized to cystectomy alone (model 4).

There was evidence of a survival benefit from chemotherapy in patients with mixed tumours ( $\mathrm{HR}=0.46 ; 95 \% \mathrm{Cl} 0.25-0.87 ; P=$ 0.02). Patients with pure UC had improved survival on the chemotherapy arm; however, the survival benefit was not statistically significant $(\mathrm{HR}=0.90 ; 95 \% \mathrm{Cl} 0.67-1.21 ; P=$ 0.48 ). There was marginal evidence that the survival benefit of chemotherapy in patients
TABLE 1 Patient characteristics for each combination of treatment with histological type

\begin{tabular}{|c|c|c|c|c|}
\hline \multirow[b]{2}{*}{ Characteristic } & \multicolumn{2}{|c|}{ Mixed tumours } & \multicolumn{2}{|l|}{ Pure UC } \\
\hline & $\begin{array}{l}\text { MVAC + } \\
\text { cystectomy }\end{array}$ & $\begin{array}{l}\text { Cystectomy } \\
\text { alone }\end{array}$ & $\begin{array}{l}\text { MVAC + } \\
\text { cystectomy }\end{array}$ & $\begin{array}{l}\text { Cystectomy } \\
\text { alone }\end{array}$ \\
\hline $\bar{N}$ & 32 & 27 & 115 & 121 \\
\hline Mean age (years) & 60 & 65 & 63 & 62 \\
\hline cT3-T4a*, \% & 59 & 70 & 59 & 57 \\
\hline Women, \% & 31 & 15 & 14 & 21 \\
\hline White, \% & 91 & 78 & 96 & 96 \\
\hline
\end{tabular}

${ }^{*}$ T3-T4a, clinical stage T3 or T4a. UC, urothelial carcinoma; MVAC, methotrexate, vinblastine, doxorubicin and cisplatin.

TABLE 2 Numbers and percentages of patients who had stage pTO at cystectomy and numbers and percentages of patients whose pTO status was not known, by treatment-histology combinations

\begin{tabular}{llccc} 
& & No. $(\%)$ & No. $(\%)$ with pT0 \\
Histological type & Treatment arm & $N$ & with pT0 & status unknown \\
\hline Mixed tumours & MVAC + cystectomy & 32 & $11(34)$ & $4(13)$ \\
Mixed tumours & Cystectomy alone & 27 & $1(4)$ & $3(11)$ \\
Pure UC & MVAC + cystectomy & 115 & $33(29)$ & $15(13)$ \\
Pure UC & Cystectomy alone & 121 & $17(14)$ & $7(6)$
\end{tabular}

UC, urothelial carcinoma; MVAC, methotrexate, vinblastine, doxorubicin and cisplatin.

TABLE 3 Estimated down-staging effects

\begin{tabular}{llrlrll} 
& Patients included & & & ADE* & \multicolumn{2}{c}{$P$} \\
Subset & in the subset & $N$ & Contrast & $\%$ & $95 \% \mathrm{Cl}, \%$ & value \\
\hline 1 & Mixed tumours & 59 & MVAC vs cystectomy-only & 28 & $(11-44)$ & 0.004 \\
2 & Pure UC & 236 & MVAC vs cystectomy-only & 15 & $(5-25)$ & 0.004 \\
3 & MVAC + cystectomy & 147 & Mixed vs pure UC & 6 & $(-11$ to 23) & 0.51 \\
4 & Cystectomy-only & 148 & Mixed vs pure UC & -8 & $(-20$ to 3) & 0.27
\end{tabular}

${ }^{*} A D E$, additive down-staging effect, directly standardized to the distribution of clinical stages among all patients included in the analysis. 95\% Cl, 95\% confidence intervals; UC, urothelial carcinoma; MVAC, methotrexate, vinblastine, doxorubicin and cisplatin.

\begin{tabular}{|c|c|c|c|c|c|c|}
\hline Model & $\begin{array}{l}\text { Patients included } \\
\text { in the model }\end{array}$ & $N$ & Contrast & $\mathrm{HR}$ & $95 \% \mathrm{Cl}$ & $\begin{array}{l}P \\
\text { value }\end{array}$ \\
\hline 1 & Mixed tumours & 59 & MVAC vs cystectomy-only & 0.46 & $(0.25-0.87)$ & 0.02 \\
\hline 2 & Pure UC & 236 & MVAC vs cystectomy-only & 0.90 & $(0.67-1.21)$ & 0.48 \\
\hline 3 & MVAC + cystectomy & 147 & Mixed vs pure UC & 0.69 & $(0.42-1.13)$ & 0.14 \\
\hline 4 & Cystectomy-only & 148 & Mixed vs pure UC & 1.28 & $(0.80-2.06)$ & 0.30 \\
\hline
\end{tabular}

$H R$, hazard ratio, adjusted for age and clinical stage; 95\% Cl, 95\% confidence intervals; UC, urothelial carcinoma; MVAC, methotrexate, vinblastine, doxorubicin and cisplatin. 
TABLE 5 Estimated five-year survival probabilities

\begin{tabular}{|c|c|c|c|c|c|}
\hline \multirow[b]{2}{*}{ Stage } & \multirow[b]{2}{*}{ Treatment } & \multicolumn{2}{|l|}{ Pure UC } & \multicolumn{2}{|l|}{ Mixed Tumors } \\
\hline & & $\overline{5-y r \text { survival }}{ }^{*}$ & $95 \% \mathrm{Cl}$ & $\overline{5 \text {-yr survival }}{ }^{*}$ & $95 \% \mathrm{Cl}$ \\
\hline$\overline{c T 2}$ & Cystectomy-only & 0.61 & $(0.52,0.72)$ & 0.54 & $(0.39,0.74)$ \\
\hline cT2 & MVAC + cystectomy & 0.64 & $(0.55,0.74)$ & 0.73 & $(0.62,0.86)$ \\
\hline сT3-T4a & Cystectomy-only & 0.42 & $(0.34,0.53)$ & 0.34 & $(0.21,0.55)$ \\
\hline сТ3-Т4а & MVAC + cystectomy & 0.46 & $(0.37,0.56)$ & 0.58 & $(0.45,0.75)$ \\
\hline
\end{tabular}

*Adjusted for age by conditional standardization (conditioned on the average age of all patients in the study); $c$ T2 = clinical stage T2, cT3-T4a = clinical stage T3-T4a. UC, urothelial carcinoma; MVAC, methotrexate, vinblastine, doxorubicin and cisplatin.

with mixed tumours was greater than it was for patients with pure UC (statistical interaction, $P=0.09$ ). These analyses also suggested that compared with pure UC, mixed tumours may be associated with increased mortality when treated with cystectomy alone and with decreased mortality when treated with MVAC plus cystectomy, although the estimated HR were relatively imprecise in these two comparisons (rows 3 and 4 of Table 4). Table 5 shows the age-standardized 5 -year survival estimates by treatment, histological type and clinical stage obtained from the Cox model. The estimated improvement in 5-year survival associated with MVAC was much greater in magnitude among patients with mixed tumours than among patients with pure UC.

All estimates in Tables 4 and 5 were controlled for age and clinical stage. These covariates were pre-specified in the original study protocol as stratification factors for survival analysis. Because the covariates gender and race also appeared to be somewhat unbalanced between the comparison subgroups (Table 1), we performed sensitivity analyses by including these covariates in the model. Gender and race were not independently associated with all-cause mortality in these analyses (after adjusting for age, clinical stage and histological type) and had no substantial impact on reported findings.

\section{DISCUSSION}

The purpose of this study was to determine whether the effect of neoadjuvant MVAC on pathological down-staging and survival of patients with locally advanced UC of the bladder treated with radical cystectomy is influenced by the presence of squamous and/ or glandular components in the tumour. This question is important because squamous and/ or glandular differentiation co-existing with malignant urothelial histology is a fairly common finding in muscle-invasive BC. To our knowledge, it has never been clearly showed that neoadjuvant PBCC can induce complete pathological response (stage pTO) and improve survival of patients with these mixed tumours. Before results of the current analyses became available, we considered it possible that the presence of non-urothelial components could render UC resistant to MVAC. If this was confirmed, then patients with mixed tumours could potentially benefit from immediate cystectomy (without neoadjuvant chemotherapy), especially as delaying cystectomy for more than 3 months from diagnosis of muscle invasion has been associated with decreased survival in some patient populations [16].

Our current analyses have showed that the presence of squamous and/or glandular differentiation does not make a locally advanced UC of the bladder resistant to neoadjuvant chemotherapy with MVAC. Evidence of tumour down-staging to pTO after chemotherapy was clearly present among patients with pure UC (ADE $=15 \%$, $P=0.004)$ and among patients with mixed tumours (ADE $=28 \%, P=0.004$ ) (Table 3 ). The actual proportions of pTOs reported in these analyses for each combination of treatment with histological type (Table 2) are slightly less than the proportions originally reported for all histological types combined (15\% in the cystectomy-only arm and 38\% in the MVAC-plus-cystectomy arm) [6]. In the original analyses, the proportions of pTOs were computed using only those patients who received cystectomy. Because some patients did not undergo cystectomy because of disease progression/unresectable disease and others refused surgery or did not receive it for unknown reasons, in the current analyses of tumour down-staging we made a conservative assumption that patients who did not undergo cystectomy, regardless of reason, had residual disease (they were considered not to have stage pTO). This is the most conservative approach to the analysis of tumour down-staging. Indeed, it is possible that some patients who refused cystectomy did so because of complete clinical response to chemotherapy. If some of these patients in fact had no residual disease, then the downstaging effect of chemotherapy reported in this paper could be underestimated. This down-staging effect (estimated under the most conservative assumptions) was nevertheless fairly large in magnitude, especially for patients with mixed tumours ( $28 \%$ on the additive scale or 28 extra pTOs per 100 patients treated, $P=0.004)$. The estimated survival benefit of chemotherapy among patients with mixed tumours was also fairly large $(H R=0.46, P=0.02)$. For patients with pure UC, the estimated improvement in survival after chemotherapy was not as substantial $(H R=0.90, P=0.48)$.

The strengths of this study include randomized treatment allocation, central pathological review (performed for most patients), the use of the standard treatment protocol (MVAC as the only form of neoadjuvant chemotherapy, no previous pelvic irradiation, etc.), and rigorous follow up. However, some limitations of this study exist. First, the results of the current analyses may be difficult or impossible to generalize to mixed urothelial tumours with non-urothelial components other than squamous cell or adenocarcinoma. For example, there is evidence to suggest that small cell tumours of the bladder may respond better to neuroendocrine regimens than to urothelial regimens such as MVAC [17]. Second, even for squamous and glandular components, survival could not be analysed separately in the current study because the estimated HR would be highly imprecise because of the small sample size for individual histological sub-types. However, our analyses suggested that pathological down-staging after chemotherapy occurs in both sub-types of mixed tumours (squamous and glandular), and clearly is not limited to only one subtype. Hence, it is unlikely that improvement in survival after chemotherapy is limited to only one of the two sub-types. 
It must also be recognized that definition and documentation of mixed histology may vary between pathologists. In our study, histological type of 50 patients was determined by institutional pathology report because slides were not available for the central review (these patients contributed 12 of the 59 mixed histology cases). Hence some potential misclassification of histological types in our study could occur, and this must be recognized as a limitation. Similarly, proportions of non-urothelial components in mixed histology tumours could not be obtained from this analysis (it was rarely reported) and so the impact of this proportion on response to MVAC or outcome in the cystectomy-only arm could not be ascertained.

Another question which needs to be considered is whether results reported in this paper are fully applicable to PBCC regimens other than MVAC. In the setting of metastatic disease, MVAC seems to result in the same survival as a less toxic regimen composed of gemcitabine and cisplatin [18]. However, the two regimens have never been directly compared in the setting of apparently resectable $\mathrm{BC}$. Hence, the ability of gemcitabine and cisplatin to induce complete pathological response and improve survival of patients with locally advanced UC of the bladder with non-urothelial components remains uncertain.

Some of the questions that could not be fully answered in this study may potentially be examined in secondary analyses of other trials of neoadjuvant PBCC for locally advanced $B C$, and possibly in pooled analyses of two or more trials. For example, a European trial of CMV (cisplatin, methotrexate and vinblastine) enrolled more than 900 patients with stage T2-T4a NO/NX MO BC [7]. To our knowledge, the effect of CMV in that trial has not been examined separately for patients with pure UC and for patients with mixed tumours. A pooled analysis of two or more trials would improve statistical power and increase the precision of estimation. This would be particularly beneficial for the formal test of statistical interaction between treatment and histological type. Unfortunately, interaction effects are difficult to detect in studies powered for the analysis of the main effects [19]. Even large interactions often produce $P$ values above $\alpha=0.05$ (the conventional level of significance for the main effects) in moderate-sized studies. Secondary analyses of larger trials and pooled analyses of two or more trials may be particularly beneficial in these situations.

In summary, presence of squamous or glandular differentiation in locally advanced UC of the bladder does not confer resistance to MVAC and in fact may be an indication for the use of neoadjuvant chemotherapy before radical cystectomy.

\section{ACKNOWLEDGEMENTS}

This investigation was supported in part by the following PHS Cooperative Agreement grant numbers awarded by the National Cancer Institute, DHHS: CA32102, CA38926, CA21115, CA35421, CA46441, CA22433, CA42777, CA58861, CA59416, CA46282, CA27057, CA14028, CA46113, CA20319, CA46136, CA45377, CA128567, CA45560, CA35431, CA32734, CA35261, CA35090, CA16385, CA58882, CA76447, CA46368, CA68183, CA28862, CA58415, CA35281, CA63844, CA35192, CA35117, CA35084, CA58686 and CA35262; and by Ashley Family Foundation.

\section{CONFLICT OF INTEREST}

None declared.

\section{REFERENCES}

1 American Cancer Society. Cancer Statistics 2009 Presentation. Available at: http://www.cancer.org/Research/Cancer FactsFigures/Cancer-Statistics-2009presentation. Accessed October 292010

2 Jemal A, Siegel R, Ward E et al. Cancer statistics 2009. CA Cancer J Clin 2009; 59 : 225-49

3 Stenzl A, Cowan NC, De Santis M et al. Guidelines on Bladder Cancer: MuscleInvasive and Metastatic. Arnheim, the Netherlands: European Association of Urology, 2008: 56

4 Stein JP, Lieskovsky G, Cote R et al. Radical cystectomy in the treatment of invasive bladder cancer: long term results in 1,054 patients. J Clin Oncol 2001; 19: 666-75

5 Advanced Bladder Cancer (ABC) MetaAnalysis Collaboration. Neoadjuvant chemotherapy in invasive bladder cancer: update of a systematic review and metaanalysis of individual patient data. Eur Urol 2005; 48: 202-5
6 Grossman HB, Natale RB, Tangen CM et al. Neoadjuvant chemotherapy plus cystectomy compared with cystectomy alone for locally advanced bladder cancer. N Engl J Med 2003; 349: 859-66

7 International Collaboration of Triallists on Behalf of the Medical Research Council Advanced Bladder Cancer Working Party. Neoadjuvant cisplatin, methotrexate, and vinblastine chemotherapy for muscle-invasive bladder cancer: a randomised controlled trial. International collaboration of trialists. Lancet 1999; 354: 533-40

8 Wasco $M$, Daignault $Y$, Zhang L et al. Urothelial carcinoma with divergent histologic differentiation (mixed histologic features) predicts the presence of locally advanced bladder cancer when detected at transurethral resection. Urology 2007; 70: 69-74

9 Logothetis CJ, Dexeus FH, Chong Cet al. Cisplatin, cyclophosphomide and doxorubicin chemotherapy for unresectable urothelial tumors: the M.D. Anderson experience. J Urol 1989; 141: 33-7

10 Kastritis E, Dimopoulos N, Antoniou C et al. The outcome of patients with advanced pure squamous or mixed squamous and transitional urothelial carcinomas following platinum-based chemotherapy. Anticancer Res 2006; 26: 3865-9

11 Beahrs $\mathrm{OH}$, Henson DE, Hutter RVP eds. Manual for Staging of Cancer, 4th edn. Philadelphia: Lippincott, 1992: 195-200

12 Rothman K, Greenland S, Lash T. Modern Epidemiology, 3rd edn. Philadelphia: Lippincott Williams \& Wilkins, 2008: 266

13 Szklo M, Nieto F. Epidemiology-Beyond the Basics, 2nd edn. Sudbury, MA: Jones and Bartlet, 2007: 422

14 Woodward M. Epidemiology - Study Design and Data Analysis. Boca Raton, FL: CRC Press, 2005: 184-5, 207

15 Cox D. Regression models and life tables. J Royal Stat Soc 1972; B34: 187

16 Gore JL, Lai J, Setodji CM et al. Mortality increases when radical cystectomy is delayed more than 12 weeks. Cancer 2009; 115: 988-96

17 Black PC, Brown GA, Colin PN et al. The impact of variant histology on the outcome of bladder cancer treated with curative intent. Urol Oncol 2009; 27: 3-7

18 Von der Maase H, Hansen SW, Roberts JT et al. Gemcitabine and cisplatin versus 
methotrexate, vinblastine, doxorubicin, and cisplatin in advanced or metastatic bladder cancer: results of a large, randomized, multinational, multicenter, phase III study. J Clin Oncol 2000; 18: 3068-77

19 Greenland S. Tests for interaction in epidemiologic studies: a review and a study of power. Stat Med 1983; 2: 243-51

Correspondence: Edward M. Messing, University of Rochester, Strong Memorial Hospital, 601 Elmwood Avenue, Box 656, Rochester, NY 14642-0001, USA. e-mail: edward_messing@urmc.rochester. edu

Abbreviations: BC, bladder cancer; PBCC, platinum-based combination chemotherapy; UC, urothelial carcinoma; SWOG, Southwestern Oncology Group; MVAC, methotrexate, vinblastine, doxorubicin, cisplatin; ADE, additive down-staging effect; $H R$, hazard ratio.

\section{EDITORIAL COMMENT}

DO MIXED HISTOLOGICAL FEATURES AFFECT SURVIVAL BENEFIT FROM NEOADJUVANT PLATINUM-BASED COMBINATION CHEMOTHERAPY IN PATIENTS WITH LOCALLY ADVANCED BLADDER CANCER?

The S8710 trial of neoadjuvant chemotherapy with methotrexate, vinblastine, adriamycin and cisplatin (M-VAC) followed by cystectomy versus cystectomy alone established the role for neoadjuvant chemotherapy in patients with muscle-invasive bladder cancer [1]. This secondary analysis of $\mathbf{S} 8710$ sought to determine if the effect seen with neoadjuvant M-VAC varied with the presence of non-urothelial components in the tumor. The presence of mixed histology in bladder tumors has been associated with aggressive disease as well as more advanced disease at the time of presentation [2,3]. For the analysis, the authors looked at nonurothelial components including squamous and/or glandular differentiation and used Cox regression models to estimate the effect of neoadjuvant chemotherapy on mortality for pure urothelial carcinoma (UC) patients and those with mixed tumors. There was an expectation that based on the previously reported aggressive nature of mixed tumors, the outcome for these patients would be inferior $[4,5]$. The analysis, however, demonstrated that patients with mixed histology tumors appear to derive a survival benefit from neoadjuvant chemotherapy $(\mathrm{HR}=0.46 ; 95 \% \mathrm{Cl}$ : $0.25-0.87 ; P=0.02$ ). Although intriguing, the interpretation of the results of this analysis is limited by several important issues. Most significant is the lack of central pathological review for 50 patient specimens where histologic type was determined from the institutional pathology reports (12 of the 59 mixed histology cases). Furthermore, the proportion of nonurothelial component in these mixed histology tumors was rarely reported. Additionally, "mixed histology" was limited to squamous and glandular differentiation and thus the analysis cannot be extended to other histologic subtypes and the small number of mixed histology cases limits the ability to detect differences in survival for the individual histologic subtypes of squamous and glandular differentiation.

Ultimately, our understanding of bladder cancer biology must not be limited to histologic variants, but must incorporate the underlying genetic and molecular drivers of disease aggressiveness as well as markers of chemosensitivity. For example, DNA repair mechanisms such as the excision repair crosscomplementation group 1 (ERCC1) in nonsmall cell lung cancer and microsatellite instability, a hallmark of the mismatch repair phenotype, in colon cancer may be used to predict for disease aggressiveness as well as for sensitivity to chemotherapy [6-8]. Host factors independent of tumor biology further confound our understanding of treatment response and outcome. As the role for pharmacogenomics such as single nucleotide polymorphisms that predict sensitivity to cisplatin are better understood, so too will our ability to tailor therapy to specific patients [9]. Ongoing studies to determine driver genetic alterations and pharmacogenomics that predict both response and resistance to therapy will undoubtedly lead to better treatment strategies and outcomes for our patients.

\section{REFERENCES}

\section{Grossman HB, Natale RB, Tangen CM} et al. Neoadjuvant chemotherapy plus cystectomy compared with cystectomy alone for locally advanced bladder cancer. N Engl J Med 2003; 349: 859-66
2 Wasco MJ, Daignault S, Zhang $Y$ et al. Urothelial carcinoma with divergent histologic differentiation (mixed histologic features) predicts the presence of locally advanced bladder cancer when detected at transurethral resection. Urology 2007; 70: 69-74

3 Domanowska $E_{1}$ Jozwicki W, Domaniewski J et al. Muscle-invasive urothelial cell carcinoma of the human bladder: multidirectional differentiation and ability to metastasize. Hum Pathol 2007; 38: 741-6

4 Logothetis CJ, Dexeus FH, Chong C et al. Cisplatin, cyclophosphamide and doxorubicin chemotherapy for unresectable urothelial tumors: the M.D. Anderson experience. JUro/ 1989; 141: 33-7

5 Kastritis E, Dimopoulos MA, Antoniou $\mathrm{N}$ et al. The outcome of patients with advanced pure squamous or mixed squamous and transitional urothelial carcinomas following platinum-based chemotherapy. Anticancer Res 2006; 26: 3865-9

6 Olaussen KA, Dunant A, Fouret $P$ et al. DNA repair by ERCC1 in non-smallcell lung cancer and cisplatin-based adjuvant chemotherapy. $N$ Engl J Med 2006; 355: 983-91

7 Lanza G, Gafa R, Santini A, Maestri I, Guerzoni L, Cavazzini L. Immunohistochemical test for MLH1 and MSH2 expression predicts clinical outcome in stage II and III colorectal cancer patients. J Clin Oncol 2006; 24 : 2359-67

8 Risio M, Reato G, di Celle PF, Fizzotti M, Rossini FP, Foa R. Microsatellite instability is associated with the histological features of the tumor in nonfamilial colorectal cancer. Cancer Res 1996; 56: 5470-4

9 O'Donnell PH, Gamazon E, Zhang W, et al. Population differences in platinum toxicity as a means to identify novel genetic susceptibility variants. Pharmacogenet Genomics 2010; 20: 327-37

\section{Arjun V. Balar ${ }^{1}$ and Matthew I. Milowsky ${ }^{1,2}$ ${ }^{1}$ Genitourinary Oncology Service, Division of Solid Tumor Oncology, Department of Medicine, Memorial Sloan-Kettering Cancer Center, New York, NY, ${ }^{2}$ Weill Cornell Medical College of Cornell University, New York,}

